\title{
Temperature dependence of the upper critical field of type-II superconductors from isothermal magnetization data. Application to high temperature superconductors
}

\author{
I. L. Landau ${ }^{1,2}$ H. R. Ott ${ }^{1}$ \\ ${ }^{1}$ Laboratorium für Festkörperphysik, ETH Hönggerberg, CH-8093 Zürich, Switzerland and \\ ${ }^{2}$ Kapitza Institute for Physical Problems, 117334 Moscow, Russia
}

(Dated: October 29, 2018)

\begin{abstract}
Using the Ginzburg-Landau theory in very general terms, we develop a simple scaling procedure which allows to establish the temperature dependence of the upper critical field $H_{c 2}$ and the value of the superconducting critical temperature $T_{c}$ of type-II superconductors from measurements of the reversible isothermal magnetization. An analysis of existing experimental data shows that the normalized dependencies of $H_{c 2}$ on $T / T_{c}$ are practically identical for all families of high- $T_{c}$ superconductors at all temperatures for which the magnetization data are available.

PACS numbers: 74.60.-w, 74.-72.-h
\end{abstract}

\section{INTRODUCTION}

Establishing the upper critical field $H_{c 2}$ and its temperature dependence from experimental data is not a simple task for high- $T_{c}$ superconductors (HTSC's). The main problem is that the transition to the superconducting state, probed by either measuring the magnetization $M(T)$ of the sample or its resistance $R(T)$, does not reveal any sharp features in $M(T)$ or $R(T)$ around $H_{c 2}$. The notorious widths of the transitions are usually attributed to fluctuation effects. One of the ways to overcome this difficulty is to use the temperature dependence of the magnetic moment of the sample $M(T)$, measured in a constant external magnetic field $H$, and to extrapolate the linear part of the $M(T)$ curve to the $M$ value corresponding to the normal state of the sample. $1-5$ This procedure is usually justified by invoking the Abrikosov theory of the mixed state according to which the magnetization per unit volume may by written as

$$
M(H, T)=\frac{1}{4 \pi} \frac{H_{c 2}(T)-H}{\left(2 \kappa^{2}-1\right) \beta_{A}},
$$

were $\kappa$ is the Ginzburg-Landau (GL) parameter and $\beta_{A}=1.16$ for a triangular vortex lattice. fU $_{\text {From Eq. }}$ (1) it follows naturally that the magnetic moment varies linearly with temperature for a fixed value of the external field, if $H_{c 2}(T)$ is a linear function of temperature. The problem is that Eq. (1) is only valid close to $H_{c 2}$. Nevertheless, most experimental $\mathrm{M}(\mathrm{T})$ curves are practically linear in $T$ for magnetic fields between $0.1 H_{c 2}$ and $0.6 H_{c 2}$, 1 i.e., well below $H_{c 2}$. In this range of magnetic fields, the magnetic moment is certainly a nonlinear function of $\left[H_{c 2}(T)-H\right]$. The apparent linearity of the experimental $M(T)$ curves is most likely the result of some non-linearity of $H_{c 2}(T)$. In this situation, a simple linear extrapolation of $M(T)$ curves will almost certainly result in wrong $H_{c 2}(T)$ curves. As an example of this type of failure, we mention a study of $\mathrm{Bi}_{2} \mathrm{Sr}_{2} \mathrm{CaCu}_{2} \mathrm{O}_{8}$ single crystals where the application of this extrapolation pracedure resulted in completely unphysical $H_{c 2}(T)$ curves.8
Another method for establishing $H_{c 2}(T)$ is to use theoretical calculations of $M(H)$ extended to $H<<H_{c 2}$ in order to evaluate $H_{c 2}$ from experimental $M(H)$ or $M(T)$ curves. This approach is again not very reliable. First of all, solving the GL equations in two dimensions for magnetic fields well below $H_{c 2}$ represents a formidable mathematical task. To our knowledge, there is only one study in which this problem has been solved numerically for the Abrikosov vortex lattice for a selected set of values of the GL parameter $\kappa$. 0 However, as far as we are aware, nobody has tried to use the results of Ref. 9 for the interpretation of the experimental data. More often, approximate models for the space dependence of the order parameter in the vortex structures are used. The most popular is the Hao-Clem model, 10 which has widely been used to derive different parameters of HTSC's from magnetization data.11-27 However, as has been poipted out recently, this model is far from being accurate.28.29 Both the Hao-Clem model and the numerical calculations in Ref. 9 assume uniform and isotropic superconductors, i.e., conditions that are definitely not met in the case of HTSC compounds.

Although the dependence of the sample resistance on temperature, $R(T)$, in external magnetic fields is often used for the evaluation of $H_{c 2}(T), 30-40$ we believe that this approach is even less reliable than the use of magnetization measurements. The transition to the normal state resistance is very gradual and there is no appropriate theory for an interpretation of $R(T)$ curves. It is quite likely that the misinterpretation of the resistance data is the main reason why $H_{c 2}(T)$ curves derived from the results of resistance measurements often exhibit an unusual positive curvature.

In order to evaluate $H_{c 2}$ from the experimental data in such complicated materials as HTSC's, it is very important to introduce an appropriate definition of the upper critical field. In an ideal type-II superconductor, $H_{c 2}$ is the highest value of a magnetic field compatible with superconductivity, i.e., the $H_{c 2}(T)$ curve on the $H-T$ phase diagram represents a line of second order phase transitions to the normal state. As is well known for HTSC superconductors, this transition degenerates to a 
cross-over region because of fluctuation effects and even in magnetic fields $H>H_{c 2}(T)$ superconducting features appear in the data of resistivity and magnetization measurements. We also note that small inclusions of another superconducting phase with a higher or lower critical temperature, $T_{c}^{\prime}$, than that of the bulk cannot always be excluded in HTSC's. In magnetic fields $H>H_{c 2}$ the impact of such inclusions with $T_{c}^{\prime}>T_{c}$ on the sample resistance or its magnetization is similar to that arising from superconducting fluctuations. At the same time, in magnetic fields well below $H_{c 2}(T)$, the effect of fluctuations and possible inclusions of impurity phases on the sample magnetization is small and the $M(H)$ curves in this magnetic field range must be practically the same as for the perfectly uniform sample without fluctuations. This circumstance provides the possibility to evaluate the temperature dependence of $H_{c 2}$, in its traditional sense, from such magnetization measurements.

In this paper we propose a new approach to this problem by scaling the $M(H)$ curves measured at different temperatures. This scaling procedure is based on the application of the GL theory, without assuming any specific magnetic field dependence of the magnetization. In this way one can only establish the temperature dependence of $H_{c 2}$, but its absolute values remain unknown. Below we describe the method in detail and apply it to experimental data available in the literature. It turns out that in many cases the extrapolation of the normalized $H_{c 2}(T)$ curve to $H_{c 2}=0$ provides reliable values of the superconducting critical temperature $T_{c}$.

\section{SCALING PROCEDURE}

Our scaling procedure is based on the assumption that the Ginzburg-Landau (GL) parameter $\kappa$ is temperature independent. Although the microscopic theory of supercenductivity predicts a temperature dependence of $\kappa, 41,22$ this dependence is rather weak and is not expected to change the results significantly. From the GL theory it follows straightforwardly that, if $\kappa$ is temperature independent, the magnetic susceptibility $\chi$ of the sample is a universal function of $H / H_{c 2}$, i.e., $\chi(H, T)=\chi(h)$ with $h=H / H_{c 2}(T)$, The magnetization density is

$$
M(H, T)=H_{c 2}(T) h \chi(h) .
$$

According to Eq. (2) the sample magnetization, for the same value of $h=H / H_{c 2}$, is proportional to $H_{c 2}(T)$. This leads to the following relation between the values of $M$ at two different temperatures, $T_{0}$ and $T$,

$$
M\left(H, T_{0}\right)=M\left(h_{c 2} H, T\right) / h_{c 2}
$$

with $h_{c 2}=H_{c 2}(T) / H_{c 2}\left(T_{0}\right)$. The collapse of individual $M(H)$ curves measured at different temperatures may be achieved by a suitable choice of $h_{c 2}(T)$. Of course, the scaling procedure implied by Eq. (3) is valid for ideal type-II superconductors only and in the following we consider the necessary corrections to Eq. (3) that are dictated by some specific features of HTSC's.

Most of the families of HTSC's reveal a weak paramagnetic susceptibility $\chi_{n}$ in the normal state. $13-19,43-46$ Its influence may be accounted for by replacing Eq. (3) by

$$
M\left(H, T_{0}\right)=M\left(h_{c 2} H, T\right) / h_{c 2}+c_{0}(T) H,
$$

where $c_{0}(T)=\chi_{n}\left(T_{0}\right)-\chi_{n}(T)$.

For many HTSC materials the derivative $d M / d H$ changes its sign when appreaching the critical temperature from below. $84-22.43-45.47-49$ Because the field dependence of the magnetization in the mixed state always requires $d M / d H>0,6$ the change of sign of $d M / d H$ cannot be explained by considering the properties of a static mixed state alone. This sign change is usually attributed to fluctuation effects. We assume that the additional contributions to the magnetization arising from fluctuation effects may be described by an effective susceptibility $\chi_{e f f}(T)$, which is independent of the applied magnetic field. In this case we can still use Eq. (4) but with $c_{0}(T)=\left[\chi_{n}\left(T_{0}-\chi_{n}(T)\right]+\left[\chi_{e f f}\left(T_{0}\right)-\chi_{e f f}(T)\right]\right.$. In the following we use the parameter $c_{0}(T)$ in Eq. (4) as an additional adjustable parameter in the scaling procedure. The assumption that $\chi_{\text {eff }}(T)$ does not depend on the magnetic field is a simplification and this is why Eq. (4) should not be used in the temperature range where $d M / d H<0$ and where the fluctuation-induced magnetization dominates the magnetic moment of the sample.

We note that the term $c_{0} H$ in Eq. (4) may also account for any contribution to the magnetization arising from small inclusions of another superconducting phase with a different $T_{c}$. If the value of $T_{c}$ of this minority phase is higher than that for the bulk of the sample, some small regions of the sample will remain in the superconducting state even if $H>H_{c 2}(T)$. These superconducting islands also give a non-zero magnetic moment with $d M / d H<0$. In magnetic fields $H<H_{c 2}$, the contribution from these regions, where superconductivity is stronger than in the bulk of the sample, is superimposed onto the contribution to the magnetic moment arising from the mixed state.

At this point, we wish to comment on the physical relevance of $H_{c 2}(T)$ and $T_{c}$ obtained in this way. Because our analysis is based on measurements of the magnetization in the mixed state, $H_{c 2}(T)$ corresponds to the disappearance of the mixed state rather than to a complete suppression of superconductivity in the sample. In magnetic fields $H>H_{c 2}$ superconducting regions may, as mentioned above, still exist in the form of separated islands formed due to either thermal fluctuations or inclusions of minority phases with enhanced values of $T_{c}$ and $H_{c 2}$. Similar superconducting islands may also exist in zero magnetic field at $T>T_{c}$. It is only important that the lateral extension of these islands is small enough, such that no mixed state can be established inside the island. The cause of such superconducting islands is not important for our consideration. It may be due to thermal fluctuations or sample inhomogeneities, as well as 
a combination of both. Note that for an ideal type-II superconductor without fluctuations these definitions of $H_{c 2}(T)$ and $T_{c}$ coincide with the values of magnetic fields and temperature fixed be the onset of superconductivity.

\section{ANALYSIS OF EXPERIMENTAL DATA}

We now apply our scaling procedure to experimental results available in the literature. As it turns out, the relative temperature variations of $H_{c 2}$ are identical for practically all HTSC materials. Because this is a completely unexpected and, in our view, rather important result, we describe the analysis in some detail. We have analyzed magnetization data for 29 samples presented in 25 publications. Some information concerning these samples is listed in Tables I to IV. Letters in the sample identification denote the chemical element characterizing the considered family of HTSC's. Because the sample homogeneity is important for the applicability of our method, only single crystals and grain-aligned samples have been chosen. We have also limited our analysis to studies in which the magnetization measurements were extended up to temperatures $T \geq 0.94-0.95 T_{c}$, because only in these cases we may expect a reliable evaluation of $T_{c}$ by extrapolating $H_{c 2}(T)$ to $H_{c 2}=0$.

In order to make use of Eq. (4), the following procedure was employed. First, the $M(H)$ curve for some temperature $T=T_{0}$ was approximated by

$$
M(H)=h_{c 2}\left\{\sum_{i=0}^{n} A_{i}\left[\ln \left(H / h_{c 2}\right)\right]^{i}+c_{0} H\right\}
$$

with $h_{c 2}=1$ and $c_{0}=0$. The coefficients $A_{i}$ were used as fit parameters and the number $n$ was chosen such that a further enhancement of its value had no influence

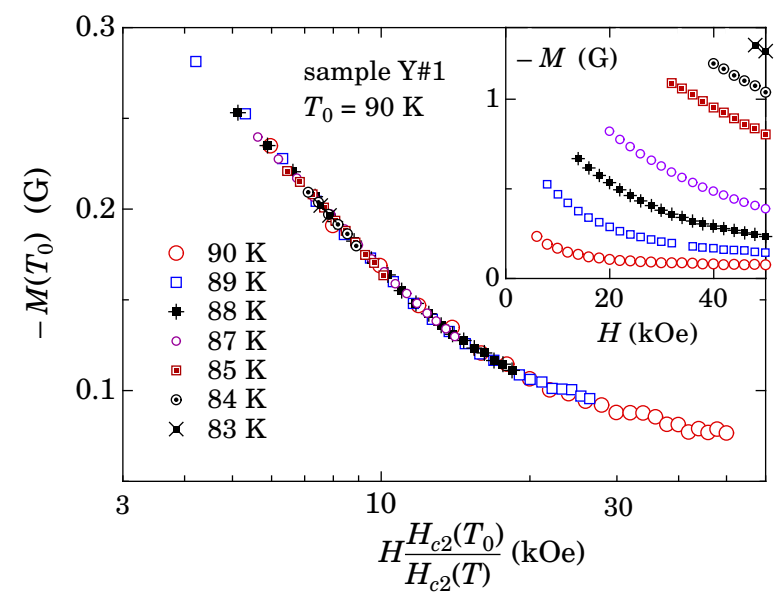

FIG. 1: The magnetization data for sample Y\# 1 after scaling using fiq. (4) with $T_{0}=90 \mathrm{~K}$. The inset displays the original data 46

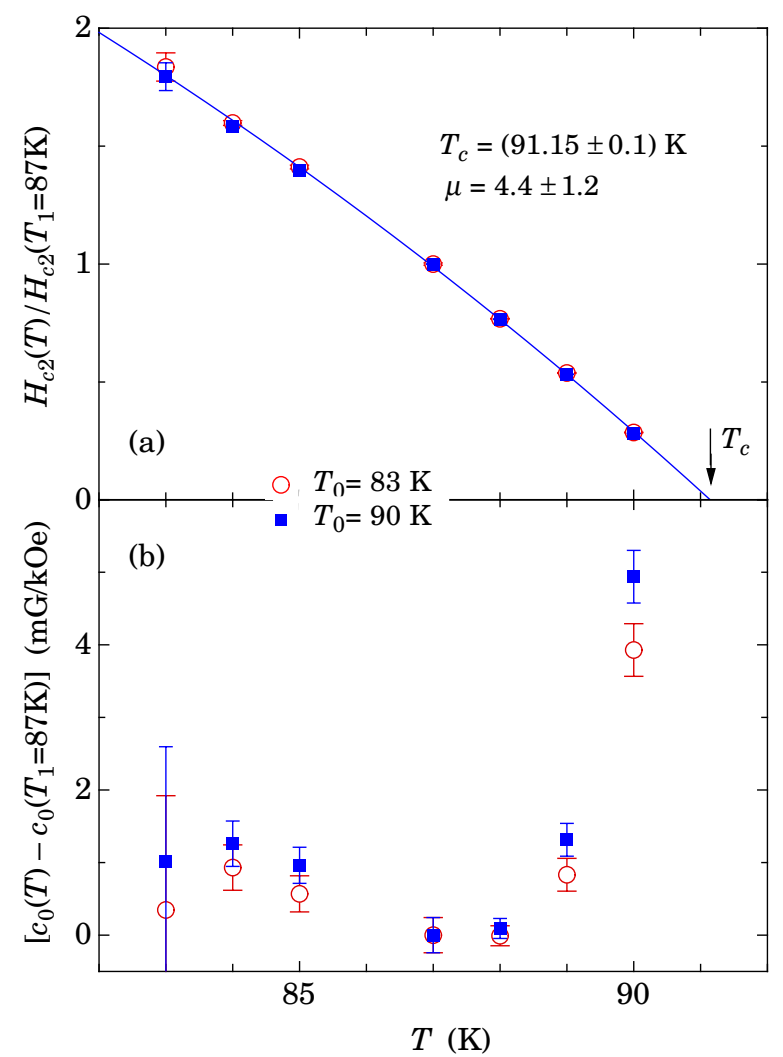

FIG. 2: (a) $H_{c 2}(T) / H_{c 2}(87 K)$ and (b) $\left[c_{0}(T)-c_{0}(87 K)\right]$ resulting for two different choices of $T_{0}$. The solid line is the best fit with Eq. (6).

on the deviation parameter $\sigma$ of the approximation of $M(H) 50$ In the next steps, the coefficients $A_{i}$ were fixed and the parameters $h_{c 2}$ and $c_{0}$ were evaluated via the fitting procedure for approximating the available $M I(H)$ curves measured at other temperatures $T=T_{i} .51$ The result of this scaling procedure, representing the field dependence of the magnetization of sample $\mathrm{Y} \# 1$ at $T=T_{0}$, is shown in Fig. 1. It may be seen that a rather perfect overlap of the individual $M(H)$ curves measured at different temperatures, which are displayed in the inset of Fig. 1, is obtained in this way. Because the renormalized field variable $h_{c 2}$ enters the denominator of Eq. (4), the magnetization data sets for the highest temperatures are considerably expanded along the vertical axis in comparison with low temperature data,. This is the reason for the somewhat enhanced scatter in the high temperature data.

In order to demonstrate the consistency of our procedure, we show typical data sets for $H_{c 2}(T)$ and $c_{0}(T)$ in Figs. 2(a) and 2(b), respectively. In this particular case, the scaling procedure was done twice, with $T_{0}$ either at the upper or at the lower end of the covered temperature range. In order to compare the results obtained in these two cases, $H_{c 2}(T)$ and $c_{0}(T)$ are normalized by their val- 

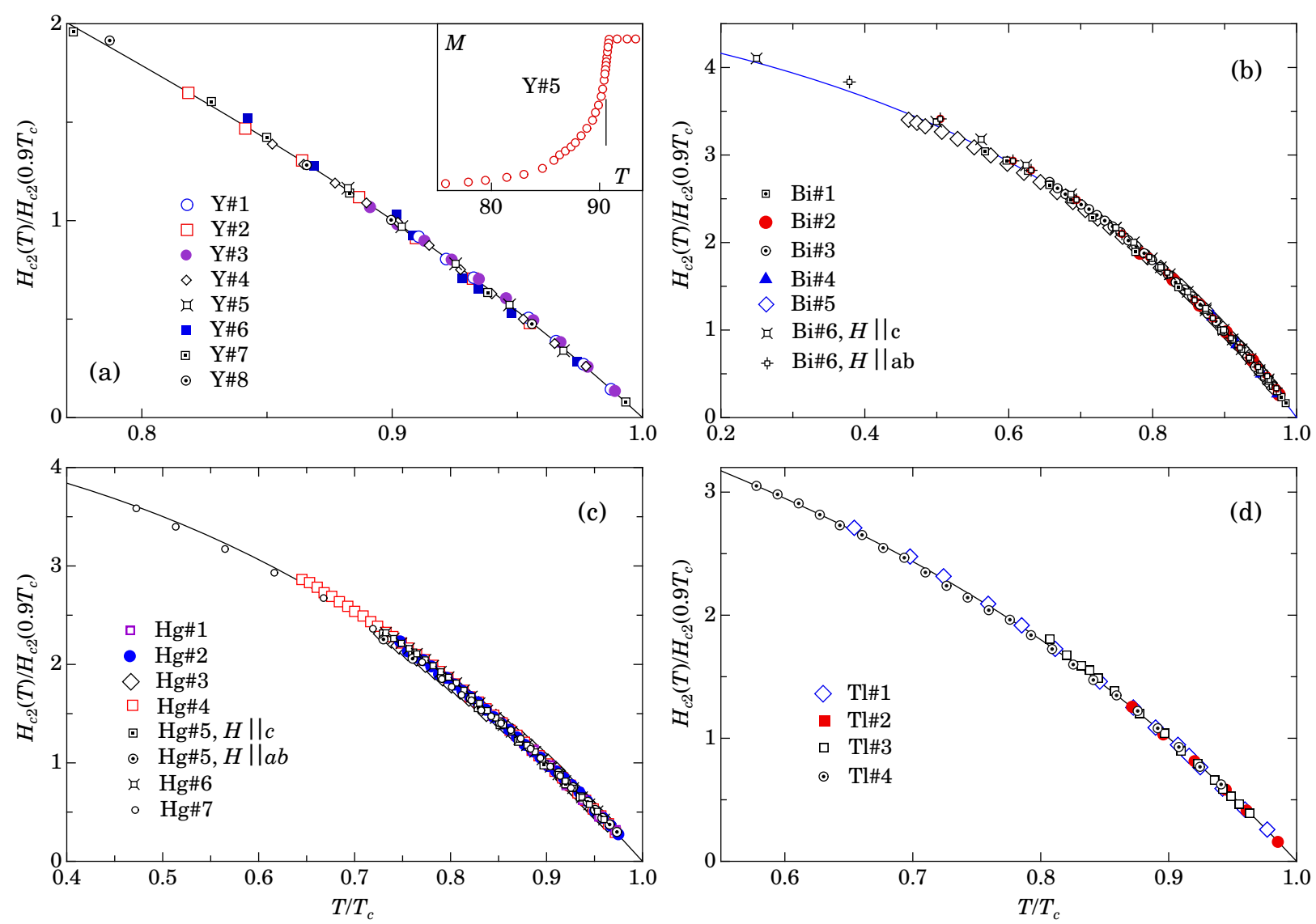

FIG. 3: $H_{c 2}(T) / H_{c 2}\left(0.9 T_{c}\right)$ versus $T / T_{c}$ for different samples. The solid lines are guides to the eye. (a) Y-based samples (Table I). The inset shows the low field $M(T)$ curve for sample Y\# 5 . The vertical line in the inset indicates the value of $T_{c}$ as obtained by extrapolation of the corresponding $h_{c 2}(T)$ curve. (b) Bi-based samples (Table II). (c) Hg-based samples (Table III). (d) Tl-based samples (Table IV)

ues at $T=T_{1}=87 \mathrm{~K}$. As may be seen, the result is practically independent of the choice of $T_{0}$. The parameter $c_{0}(T)$ in Eq. (4) accounts for only a small correction to $M(H)$. This causes a much enhanced uncertainty in the values of $c_{0}(T)$ than that for the normalized upper critical field, as may easily be seen by comparing Figs. 2(a) and 2(b).

We note that the uncertainty of $h_{c 2}(T)$ increases considerably for temperatures close to $T_{c}$ as well as for the lowest temperatures. The loss of accuracy for the highest temperatures is due to the obvious enhancement of the experimental uncertainty of the $M(H)$ data. Although the accuracy is improving with decreasing temperature, the increase of the irreversibility field limits the available magnetic field range as may clearly be seen in the inset to Fig. 1. If the experimental data are collected in a narrow magnetic field range only, our scaling procedure is not reliable.

The temperature dependence of the normalized upper critical field, as shown in Fig. 2(a), may also be used to evaluate the critical temperature $T_{c}$. For this purpose the ratio $H_{c 2}(T) / H_{c 2}\left(T_{1}\right)$ was approximated by

$$
\frac{H_{c 2}(T)}{H_{c 2}\left(T_{1}\right)}=\frac{1-\left(T / T_{c}\right)^{\mu}}{1-\left(T_{1} / T_{c}\right)^{\mu}},
$$

in which $\mu$ and $T_{c}$ are used as fit parameters. Eq. (6) provides a rather good approximation to $h_{c 2}(T)$ curves for $T \geq 0.8 T_{c}$. The corresponding fit is shown as the solid line in Fig. 2(a). The values of $\mu$ and $T_{c}$ are indicated in Fig. 2.52 If the experimental data were obtained up to temperatures rather close to the critical temperature, the extrapolated value of $T_{c}$ is quite accurate. A reliable value of $T_{c}$ is essential for the comparison of the results that were obtained for the samples with different critical temperatures. Using the values of $T_{c}$ evaluated in such a way, we have plotted $H_{c 2}(T) / H_{c 2}\left(0.9 T_{c}\right)$ versus $T / T_{c}$ as shown in Fig. 3(a). Quite surprisingly, the temperature variations of $\mathrm{H}_{c 2}$ for different Y-based compounds and different types of samples turn out to be identical. In the inset of Fig. 3(a) we display the low field magnetization curve $M(T)$ of sample Y\#5 and indicate the position of $T_{c}$ resulting from our extrapolation procedure with a 


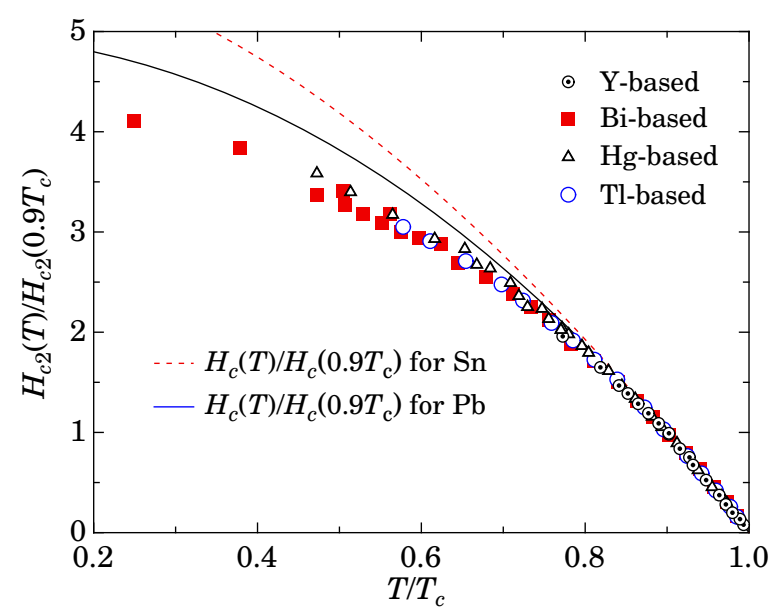

FIG. 4: The normalized temperature dependence of $H_{c 2}$ for different HTSC compounds. The solid and broken lines represent the ratios $H_{c}(T) / H_{c}\left(0.9 T_{c}\right)$ for pure metallic Lead and Tin, respectively.

vertical line.

The temperature variations of $h_{c 2}$ for other families of HTSC's are plotted in Figs. 3(b) - 3(d). Similar to what has been found for Y-based compounds, the scaling procedure again leads to an almost perfect merging of all the data onto one single curve for different samples. Furthermore, as may clearly be seen in Fig. 4, the temperature dependencies of the normalized upper critical field for different families of HTSC's are practically identical at all temperatures for which the experimental data are available. We note that the insignificant differences between the $h_{c 2}\left(T / T_{c}\right)$ curves for different samples, visible at the lowest temperatures in Figs. 3(a), 3(b), and 4, are due to small errors in the determination of the critical temperature. For the data presented in Figs. 3(a-d) the relative errors in the determination of the critical temperature, $\Delta T_{c} / T_{c}$, are between 0.001 and 0.003 , depending on the quality of the original experimental data. Although this uncertainty is quite small, it is sufficient to explain the observed differences between the $h_{c 2}$ values at low temperatures.

Among the numerous samples listed in Tables I - IV, only for the oxygen deficient sample Y\#9, the $h_{c 2}(T)$ curve is distinctly different. As may be seen in Fig 5, $h_{c 2}(T)$ for this sample is perfectly linear in the entire covered temperature range, in striking difference to two other, over-doped and optimally doped Y-based samples (Y\#7 and Y\#8) investigated in the same study. The magnetization data for sample $\mathrm{Y} \# 9$ were collected in a very wide range of magnetic fields and, as may be seen in Fig. 6, our scaling leads again to a nearly perfect merging of the curves. The difference in $h_{c 2}(T)$ between Y\#9 and other samples is thus not due to insufficient sample quality but rather reflects the intrinsic difference in properties of under-doped $\mathrm{YBa}_{2} \mathrm{Cu}_{3} \mathrm{O}_{7-x}$ materials.



FIG. 5: $H_{c 2}(T) / H_{c 2}\left(0.9 T_{c}\right)$ data for several $\mathrm{YBa}_{2} \mathrm{Cu}_{3} \mathrm{O}_{7 x}$ samples with different oxygen contents. The solid line represents $H_{c 2}(T) / H_{c 2}\left(0.9 T_{c}\right)$ for the Y-based samples shown in Fig. 3(a). The dashed line is the best fit to the data for sample $\mathrm{Y} \# 9$, assuming a linear temperature variation of $H_{c 2}$.

Only very few magnetization studies of oxygen deficient $\mathrm{YBa}_{2} \mathrm{Cu}_{3} \mathrm{O}_{7-x}$ single crystals or grain-aligned samples are available in the literature and we could find only two additional publications which are suitable for our analysis (samples Y\#10 and Y\#11). 5 .53 Unfortunately, as may be seen in Fig. 5, the measurements reported in Refs. 5 and 53 were made at temperatures very close to $T_{c}$ and in this temperature range, the temperature dependence of $H_{c 2}$ is linear for all HTSC materials.

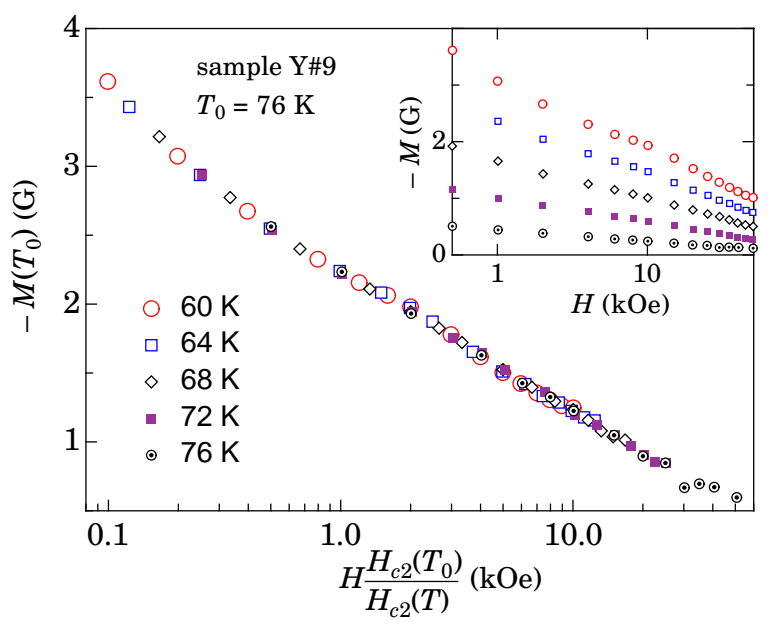

FIG. 6: The magnetization data for sample Y\# 9 after scaling using-Fq. (4) with $T_{0}=76 \mathrm{~K}$. The inset displays the original data 11

HTSC's are strongly anisotropic and it is well known that, depending on the orientation of the applied mag- 


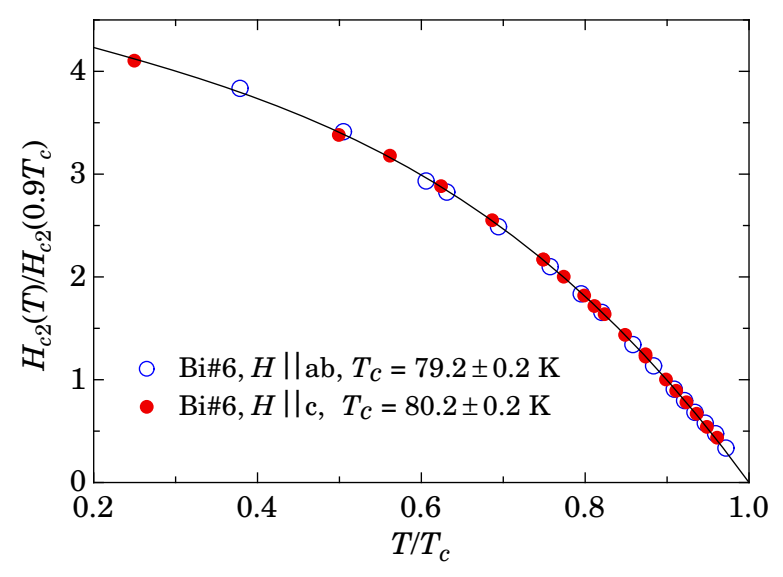

FIG. 7: $H_{c 2}(T) / H_{c 2}\left(0.9 T_{c}\right)$ data for sample Bi\# 8 for 2 different orientations of the external magnetic field.

netic field, the absolute values of $H_{c 2}$ differ significantly. This is why it is interesting to compare the results of our analysis for different orientations of the magnetic field. Unfortunately, we have found only two data sets from magnetization measurements that were made on the same samples but with two different orientations of the external magnetic field $(\mathrm{Hg} \# 5$ and $\mathrm{Bi} \# 6)$. As may be seen in Fig. 3(c), the results for the grain-aligned sample $\mathrm{Hg} \# 5$ are practically independent of the orientation of the magnetic field. The situation for the single-crystal sample Bi\#6 is different. The resulting $h_{c 2}(T)$ curves for this sample are shown in Fig. 7. The data perfectly match each other if we assume that the value of $T_{c}$ depends on the orientation of the magnetic field. This at first glance rather strange result may easily be understood if we recall our definition of $H_{c 2}$ at the end of Section 2 and we discuss this point in the next paragraph.

As has been described above, our procedure provides $H_{c 2}$ as it enters the equation for the magnetization in the mixed state, i.e., the resulting $H_{c 2}(T)$ curve in the $H-T$ phase diagram represents the upper boundary for the existence of the mixed state. In a perfect type-II superconductor without fluctuations this definition of $H_{c 2}$ coincides with the upper limit for the existence of superconductivity. In real HTSC materials the situation is different and superconducting regions may still exist in the sample even above the $H_{c 2}(T)$ curve due to, for instance, thermal fluctuations or impurities with a higher $T_{c}$. We consider it as an advantage that such effects have practically no influence on our evaluation of $H_{c 2}$. The situation is further complicated by the layered structure of HTSC's. As has previously been established by resistance measurements in zero magnetic field for Bi-based compounds, the superconducting coherence in the $\mathrm{Cu}-\mathrm{O}$ planes sets in at a somewhat higher temperature than glong the direction perpendicular to the planes.54.55.56.5758 The same conclusion can be gained from results of magnetization measurements in magnetic fields of several Oersteds 47.59, 60 This justifies the introduction of two critical temperatures $T_{c}^{(a b)}$ and $T_{c}^{(c)}$. Below $T_{c}^{(a b)}$, the superconducting phase coherence is established along the $a b$-planes, but only below $T_{c}^{(c)}$ supercurrents can propagate in the direction of the $c$-axis. In the temperature range $T_{c}^{(c)}<T<T_{c}^{(a b)}$, although superconductivity already exists in the $a b$-planes, no mixed state can be created in magnetic fields parallel to the planes. This occurs only at $T \leq T_{c}^{(c)}$, i.e., in this case our evaluation of $T_{c}$ corresponds to $T_{c}^{(c)}$. If the magnetic field is parallel to the $c$-axis, the mixed state can be created already at $T_{c}^{(a b)}$. This simple picture gives a natural explanation for the difference in $T_{c}$ for the different orientations of the magnetic field that was obtained for the sample Bi\#6 (see Fig. 7). The observed difference in $T_{c}$ is quite small $\left(\Delta T_{c} / T_{c} \approx 0.01\right)$ and may easily be masked, for instance, by grain misalignments in grainaligned samples. This could be the reason why we do not see this effect in the sample $\mathrm{Hg} \# 4$. It is also possible that this difference in $T_{c}$ is of significant magnitude only in Bi-based cuprates due to their very special crystalline structure.

\section{DISCUSSION}

The scaling procedure based on Eq. (4) turns out to be rather successful for the analysis of the reversible magnetization of HTSC's. Figs. 1 and 6 demonstrate very well the scaling of isothermal magnetization data resulting in plots of the magnetization at a chosen temperature versus a renormalized magnetic field. The quality of scaling is remarkable for all cases that are listed in Tables I-IV and the mismatch between the $M(H)$ curves measured at different temperatures does not exceed the scatter of the original experimental data.

The most surprising result of our analysis is that for practically all families of HTSC's the $h_{c 2}\left(T / T_{c}\right)$ curves are virtually identical (Fig. 4). It is difficult to imagine that this universality of the $h_{c 2}\left(T / T_{c}\right)$ dependence is just a coincidence. We are of the opinion that the spectacular agreement between the $h_{c 2}\left(T / T_{c}\right)$ data for a great variety of different samples is an unambiguous evidence that our approach captures the essential features of the magnetization process of HTSC's. It does not necessarily mean, of course, that the Ginzburg-Landau parameter $\kappa$ is indeed temperature independent. The universality of $h_{c 2}\left(T / T_{c}\right)$ is preserved if the temperature dependence of $\kappa$ is the same for the different HTSC compounds studied here.

Our analysis is applicable only to reversible magnetization data and therefore, all the results and conclusions are limited to temperatures close to $T_{c}$. The lower limit of validity, $T_{\min }$, is quite different for different families of HTSC's, as may be seen in Fig. 4. The ratio $T_{\min } / T_{c}$, which depends on the strength of the pinning of vortices, 
is highest for the Y-based compounds that exhibit the strongest pinning forces.

The universality of the normalized temperature dependence of $H_{c 2}$ implies that the normalized temperature variations of the thermodynamic critical field, $H_{c}$ for different HTSC's are also identical. Since $H_{c}^{2} / 8 \pi$ is the difference in the free energy densities between the normal and superconducting states, $H_{c}(T)$ also reflects the temperature dependence of the superconducting energy gap $\Delta .9$ In other words, our result that the normalized temperature dependence of $H_{c 2}$ follows the same universal curve for different families of HTSC's implies that the normalized temperature variations of the energy gap $\Delta\left(T / T_{c}\right) / \Delta(0)$ for different HTSC's are also identical, at least in the temperature ranges covered in this study.

We note that the temperature dependencies of $H_{c 2}$ for HTSC's obtained as a result of our analysis are qualitatively very similar to those of conventional superconductors. They are linear at temperatures close to $T_{c}$ with a pronounced negative curvature at lower temperatures. Apparently, the positive curvature of $H_{c 2}(T)$ for HTSC's, which is often reported in the literature, is due to the uncertainty of defining $\mathrm{H}_{c 2}$ in those studies.

\section{CONCLUSION}

We have developed a scaling procedure that allows to obtain the temperature dependence of the upper critical field from the measurements of the reversible isothermal magnetization. If the magnetization measurements are extended up to temperatures close to the superconducting critical temperature, our procedure also allows for a fairly reliable evaluation of the zero-field critical temperature. We have applied this scaling procedure for the analysis of experimental data for high- $T$ superconductors available in the literature and have shown that the normalized temperature dependencies of $H_{c 2}$ are qualitatively the same as those of conventional superconductors and we obtain the same universal curve for different families of HTSC's. This universality is a very strong indication that also the temperature dependence of the superconducting energy gap is the same for all cuprate superconductors. All these statements have been verified to be valid at all temperatures for which data of measurements of the reversible magnetization of different types of cuprate superconductors are available in the literature.
TABLE I: Sample identification of Y-based materials.

\begin{tabular}{ccccc}
\hline No. & Refs. & $\mathrm{Compound}_{2}$ & Sample & $T_{c}(\mathrm{~K})$ \\
\hline $\mathrm{Y} \# 1$ & 46 & $\mathrm{YBa}_{2} \mathrm{Cu}_{3} \mathrm{O}_{7-x}$ & single crystal & 91.1 \\
$\mathrm{Y} \# 2$ & 61 & $\mathrm{YBa}_{2} \mathrm{Cu}_{3} \mathrm{O}_{7}$ & single crystal & 88.0 \\
$\mathrm{Y} \# 3$ & 17 & $\mathrm{YBa}_{2} \mathrm{Cu}_{3} \mathrm{O}_{7-x}$ & grain-aligned & 92.0 \\
$\mathrm{Y \# 4}$ & 16 & $\mathrm{YBa}_{2} \mathrm{Cu}_{4} \mathrm{O}_{8}$ & grain-aligned & 79.8 \\
$\mathrm{Y} \# 5$ & 62 & $\mathrm{YBa}_{2} \mathrm{Cu}_{3} \mathrm{O}_{7-x}$ & single crystal & 93.0 \\
$\mathrm{Y} \# 6$ & 63 & $\left(\mathrm{YCa}^{2} \mathrm{~Pb}_{2} \mathrm{Sr}_{2} \mathrm{Cu}_{3} \mathrm{O}_{8+x}\right.$ & single crystal & 76.0 \\
$\mathrm{Y} 7$ & 11 & $\mathrm{YBa}_{2} \mathrm{Cu}_{3} \mathrm{O}_{6.94}$ & grain-aligned & 92.9 \\
$\mathrm{Y} \# 8$ & 11 & $\mathrm{YBa}_{2} \mathrm{Cu}_{3} \mathrm{O}_{7}$ & grain-aligned & 88.7 \\
$\mathrm{Y} \# 9$ & 11 & $\mathrm{YBa}_{2} \mathrm{Cu}_{3} \mathrm{O}_{6.85}$ & grain-aligned & 79.9 \\
$\mathrm{Y} \# 10$ & 5 & $\mathrm{YBa}_{2} \mathrm{Cu}_{3} \mathrm{O}_{6.65}$ & single crystal & 62.3 \\
$\mathrm{Y \# 11}$ & 53 & $\mathrm{YBa}_{2} \mathrm{Cu}_{3} \mathrm{O}_{6.5}$ & single crystal & 44.8 \\
\hline
\end{tabular}

TABLE II: Sample identification of Bi-based materials.

\begin{tabular}{ccccc}
\hline No. & Refs. & Compound & Sample & $T_{c}(\mathrm{~K})$ \\
\hline $\mathrm{Bi} \# 1$ & 64 & $\mathrm{Bi}_{2} \mathrm{Sr}_{2} \mathrm{Ca}_{2} \mathrm{Cu}_{2} \mathrm{O}_{8+x}$ & single crystal & 84.0 \\
$\mathrm{Bi} \# 2$ & 65 & $(\mathrm{BiPb})_{2} \mathrm{Sr}_{2} \mathrm{Ca}_{2} \mathrm{Cu}_{3} \mathrm{O}_{x}$ & whisker & 108.7 \\
$\mathrm{Bi \# 3}$ & 26 & $(\mathrm{BiPb})_{2} \mathrm{Sr}_{2} \mathrm{Ca}_{2} \mathrm{Cu}_{2} \mathrm{O}_{8}$ & single crystal & 91.4 \\
$\mathrm{Bi \# 4}$ & 48 & $\mathrm{Bi}_{2} \mathrm{Sr}_{2} \mathrm{Ca}_{2} \mathrm{Cu}_{2} \mathrm{O}_{8}$ & single crystal & 88.2 \\
$\mathrm{Bi \# 5}$ & 66 & $\mathrm{Bi}_{2.1} \mathrm{Sr}_{1.7} \mathrm{Ca}_{1.2} \mathrm{Cu}_{2} \mathrm{O}_{x}$ & single crystal & 86.7 \\
$\mathrm{Bi \# 6}$ & 8 & $\mathrm{Bi}_{2} \mathrm{Sr}_{2} \mathrm{Ca}_{2} \mathrm{Cu}_{2} \mathrm{O}_{8}$ & single crystal & 80.5 \\
$\mathrm{Bi \# 6}$ & 8 & $\mathrm{Bi}_{2} \mathrm{Sr}_{2} \mathrm{Ca}_{2} \mathrm{Cu}_{2} \mathrm{O}_{8}$ & single crystal & 79.1 \\
\hline
\end{tabular}

TABLE III: Sample identification of Hg-based materials.

\begin{tabular}{ccccc}
\hline No. & Refs. & Compound & Sample & $T_{c}(\mathrm{~K})$ \\
\hline $\mathrm{Hg} \# 1$ & 67 & $\mathrm{HgBa}_{2} \mathrm{CaCu}_{2} \mathrm{O}_{6+x}$ & grain-aligned & 117.1 \\
$\mathrm{Hg} \# 2$ & 68 & $\mathrm{HgBa}_{2} \mathrm{Ca}_{2} \mathrm{Cu}_{4} \mathrm{O}_{10+x}$ & grain-aligned & 123.1 \\
$\mathrm{Hg} \# 3$ & 24 & $\mathrm{Hg}_{0.7} \mathrm{~Pb}_{0.3} \mathrm{Sr}_{2} \mathrm{Ca}_{2} \mathrm{Cu}_{3} \mathrm{O}_{x}$ & grain-aligned & 125.5 \\
$\mathrm{Hg} \# 5$ & 13 & $\mathrm{HgBa}_{2} \mathrm{Ca}_{2} \mathrm{Cu}_{3} \mathrm{O}_{8+x}$ & grain-aligned & 131.5 \\
$\mathrm{Hg} \# 5$ & 13 & $\mathrm{HgBa}_{2} \mathrm{Ca}_{2} \mathrm{Cu}_{3} \mathrm{O}_{+}+\mathrm{x}$ & grain-aligned & 131.5 \\
$\mathrm{Hg} \# 6$ & 21 & $\mathrm{Hg}_{1-y} \mathrm{~Pb}_{y} \mathrm{Ba}_{2-z} \mathrm{Sr}_{z} \mathrm{Ca}_{2} \mathrm{Cu}_{3} \mathrm{O}_{x}$ & grain-aligned & 124.6 \\
$\mathrm{Hg} \# 7$ & 71 & $\left(\mathrm{HgCu} \mathrm{Ba}_{2} \mathrm{CuO}_{4+x}\right.$ & single crystal & 97.4 \\
\hline
\end{tabular}


TABLE IV: Sample identification of Tl-based materials.

\begin{tabular}{ccccc}
\hline No. & Refs. & Compound & Sample & $T_{c}(\mathrm{~K})$ \\
\hline $\mathrm{Tl} \# 1$ & 15 & $\mathrm{Tl}_{2} \mathrm{Ba}_{2} \mathrm{Ca}_{2} \mathrm{Cu}_{3} \mathrm{O}_{10}$ & grain-aligned & 114.6 \\
$\mathrm{Tl} \# 2$ & 69 & $\mathrm{Tl}_{2} \mathrm{Ba}_{2} \mathrm{Ca}_{2} \mathrm{Cu}_{3} \mathrm{O}_{10+x}$ & grain-aligned & 122.8 \\
$\mathrm{Tl} \# 3$ & 70 & $\mathrm{Tl}_{0.5} \mathrm{~Pb}_{0.5} \mathrm{Sr}_{2} \mathrm{CaCu}_{2} \mathrm{O}_{7}$ & single crystal & 76.9 \\
$\mathrm{Tl} \# 4$ & 25 & $\mathrm{TlBa}_{2} \mathrm{Ca}_{3} \mathrm{Cu}_{4} \mathrm{O}_{11+x}$ & single crystal & 121.2 \\
\hline
\end{tabular}

1 U. Welp, W. K. Kwok, G. W. Crabtree, K. G. Vandervoort, and J. Z. Liu, Phys. Rev. Lett. 62, 1908 (1989).

${ }^{2}$ U. Welp, W. K. Kwok, G. W. Crabtree, K. G. Vandervoort, and J. Z. Liu, Phys. Rev. B 40, 5263 (1989).

3 U. Welp, M. Grmsditch, H. You, W. K. Kwok, M. M. Fang, G. W. Crabtree, and J. Z. Liu, Physica C 161, 1 (1989).

${ }^{4}$ U. Welp, M. Grmsditch, H. You, W. K. Kwok, M. M. Fang, G. W. Crabtree, and J. Z. Liu, Physica B 163, 473 (1990).

${ }^{5}$ K. G. Vandervoort, U. Welp, J. E. Kessler, H. Claus, G. W. Crabtree, W. K. Kwok, A. Umezawa, B. W. Veal, J. W. Downey, A. P. Paulikas, and J. Z. Liu, Phys. Rev. B 43, 13042 (1991).

6 A. A. Abrikosov, Zh. Eksp. Teor. Fiz. 32, 1442 (1957) [Sov. Phys. JETP 5, 1174 (1957)].

7 A. A. Abrikosov, Fundamentals of the theory of metals. North-Holland, Amsterdam, 1988.

${ }^{8}$ W. Kritscha, F. M Sauerzopf, H. W. Weber, G. W. Garbtree, Y. C. Chang, and P. Z. Jiang, Physica C 179, 59 (1991).

9 E. H. Brandt, Phys. Rev. Lett. 78, 2208 (1997).

10 Z. Hao and J. R. Clem, M. W. McElfresh, L. Civale, A. P. Malozemoff, and F. Holtzberg, Phys. Rev. B 43, 2844 (1991).

11 J. G. Ossandon, J. R. Thompson, D. K. Christen, B. C. Sales, H. R. Kerchner, J. O. Thomson, Y. R. Sun, K. W. Lay, and J. E. Tkaczyk, Phys. Rev. B 45, 12534 (1992).

12 Qiang Li, M. Suenaga, T. Kimura, and K.Kishio, Phys. Rev. B 47, 11384 (1993).

13 Y. C. Kim, J. R. Thompson, J. G. Ossandon, D. K. Christen, and M. Paranthaman, Phys. Rev. B 51, 11767 (1995).

14 U. Welp, S. Fleshler, W. K. Kwok, J. Downey, G. W. Crabtree, H. Claus, A. Erb, and G. Müller-Vogt, Phys. Rev. B 47, 12369 (1993).

15 D. N. Zheng, A. M. Campbell, and R. S. Liu, Phys. Rev. B 48, 6519 (1993).

16 Junghyun Sok, Ming Xu, Wei Chen, B. J. Suh, J. Gohng, D.K. Finnemore, M.J. Kramer, L.A. Schwartzkopf, and B. Dabrowski, Phys. Rev. B 51, 6035 (1995).

17 Junho Gohng and D. K. Finnemore, Phys. Rev. B 46, 398 (1992).

18 D. N. Zheng, A. M. Campbell, and R. S. Liu, Phys. Rev. B 48, 6519 (1993).

19 Ming Xu, Junghyun Sok, J. E. Ostenson, D. K. Finnemore, and B. Dabrowski, Phys. Rev. B 53, 15313 (1996).

20 Mung-Seog Kim, Sung-Ik Lee, Seong-Cho Yu, and Nam H. Hur, Phys. Rev. B 53, 9460 (1996).

21 Yi Zhuo, Jae-Hyuk Choi, Mung-Seog Kim, Wan-Seon Kim, Z. S. Lim, Sung-Ik Lee, and Sergey Lee, Phys. Rev. B 55,
12719 (1997).

${ }^{22}$ Yi Zhuo, Jae-Hyuk Choi, Mung-Seog Kim, Jin-Nam Park, Myoung-Kwang Bae, and Sung-Ik Lee, Phys. Rev. B 56, 8381 (1997).

23 J. R. Thompson, J. G. Ossandon, D. K. Christen, M. Paranthaman, E. D. Specht, and Y. C. Kim, Phys. Rev. B 54, 7505 (1996).

24 Yi Zhuo, Su-Mi Oh, Jae-Hyuk Choi, Mun-Seog Kim, SungIk Lee, N. P. Kiryakov, M. S. Kuznetsov, and Sergey Lee, Phys. Rev. B 60, 13094 (1999).

${ }^{25}$ Lu Zhang, J. Z. Liu, and R. N. Shelton, Solid State Comm. 109, 761 (1999).

${ }^{26}$ Lu Zhang, J. Z. Liu, and R. N. Shelton, Phys. Rev. B 45, 4978 (1992).

27 A. A. Nugroho, I. M. Sutjahja, A. Rusydi, M. O. Tjia, A. A. Menovsky, F. R. de Boer, and J. J. M. Franse, Phys. Rev. B 60, 15384 (1999).

28 W. V. Pogosov, K. I. Kugel, and A. L. Rakhmanov, Zh. Eksp. Teor. Fiz. 118, 676 (2000) [JETP 91, 588 (2000)].

29 W. V. Pogosov, K. I. Kugel, A. L. Rakhmanov, and E. H. Brandt, Phys. Rev. B 64, 064517 (2001).

30 T. T. M. Palstra, B. Batlogg, L. F. Schneemeyer, R. B. van Dover, and J. V. Waszczak, Phys. Rev. B 38, 5102 (1988).

31 M. J. Naughton, R. C. Yu, P. K. Davies, J. E. Fischer, R. V. Chamberlin, Z. Z. Wang, T. W. Jing, N. P. Ong, and P. M. Chaikin, Phys. Rev. B 38, 9280 (1988).

32 Ichiro Matsubara, Hideo Tanigawa, Toru Ogura, Hiroshi Yamashita, Makoto Kinoshita, and Tomoji Kawai, Phys. Rev. B 45, 7414 (1992).

33 A. S. Alexandrov, V. N. Zavaritsky, W. Y. Liang, and P. L. Nevsky, Phys. Rev. Lett 76, 983 (1996).

34 X.-J. Xu, L. Fu, and Y.-H. Zhang, Physica C 282-287, 1557 (1997).

35 M. N. Khlopkin, G. Kh. Panova, N. A. Chernoplekov, A. A. Shikov, and A. V. Suetin, Zh. Eksp. Teor. Fiz. 112, 1386 (1997) [JETP 85, 755 (1997)].

${ }^{36}$ V. F. Gantmakher and G. E. Tsydynzhapov, Zh. Eksp. Teor. Fiz. 115, 268 (1999) [JETP 88, 148 (1999).

37 S. I. Vedeneev, A. G. M. Jansen, E. Haanappel, and P. Wyder, Phys. Rev. B 60, 12467 (1999).

38 Yoichi Ando, G. S. Boebinger, A. Passner, L. F. Schneemeyer, T. Kimura, M. Okuya, S. Watauchi, J. Shimoyama, K. Kishio, K. Tamasaku, N. Ichikawa, and S. Uchida, Phys. Rev. B 60, 12475 (1999).

39 V. F. Gantmakher, G. A. Emel'chenko, I. G. Naumenko, and G. E. Tsydynzhapov, Pisma Zh. Eksp. Teor. Fiz. 72, 33 (2000) [JETP Letters 72, 21 (2000)].

${ }^{40}$ V. N. Zavaritsky, V. V. Kabanov, and A. S. Alexandrov, 
cond-mat/0204012

41 L. P. Gor'kov, Zh. Eksp. Teor. Fiz. 37, 833 (1959) [Soviet Phys.-JETP 10, 593 (1960)].

42 E. Helfand and N. R. Werthamer, Phys Rev. 147, 288 (1966).

43 Yung M. Huh, J. E. Ostenson, F. Borsa, V. G. Kogan, D. K. Finnemore, A. Vietkin, A. Revcolevschi, M.-H. Julien, Phys. Rev. B 63, 064512 (2001).

44 O. Jeandupeux, A. Schilling,H. R. Ott, and A. vanOtterlo, Phys. Rev. B 53, 12475 (1996).

45 Qiang Li, K. Shibutani, M. Suenaga, I. Shigaki, and R. Ogawa, Phys. Rev. B 48, 9877 (1993).

46 J. R. Cooper, J. W. Loram, J. D. Johnson, J. W. Hodby, and Chen Changkang, Phys. Rev. Lett. 79, 1730 (1997).

47 G. Le Bras, L. Fruchter, V. Vulcanescu, V. Viallet, A. Bertinotti, A. Forget, J. Hammann, J.-F. Marucco, and D. Colson, Physica C 271, 205 (1996).

48 E. G. Miramontes, J. A. Campá, A. Pomar, I. Rasines, C. Torrón, J. A. Veira, and Félix Vidal, Physica C 235-240, 2931 (1994).

49 R. Jin, A. Schilling, and H. R. Ott, Phys. Rev. B 49, 9218 (1994).

50 This function has been chosen only because it fits the experimental $M(H)$ data quite well and it may be replaced by any other suitable function.

51 The measurements at different temperatures usually cover different ranges of the normalized magnetic field $H / h_{c 2}$. This is why it is important that only those data points at $T=T_{i}$, for which the values of $H / h_{c 2}$ are inside the magnetic field range covered at $T=T_{0}$, are considered in the fitting procedure.

52 The error margins indicated in this paper are solely due to the approximation. Taking into account experimental errors may enhance the error margins considerably.

53 B. Rosenstein, B. Ya. Shapiro, R. Prozorov, A. Shaulov, and Y. Yeshurun, Phys. Rev. B 63, 134501 (2001).

54 G. Briceño, M. F. Crommie, and A Zettl, Phys. Rev. Lett. 66, 2164 (1991).

55 J. H. Cho, M. P. Maley, S. Fleshler, A. Lacerda, and L. N.
Bulaevskii, Phys. Rev. B 50. 6493 (1994).

56 Xiao-Guang Li, Xuefeng Sun, Wenbin Wu, Qianwang Chen, Lei Shi, Yuheng Zhang, Y. Kotaka, and K. Kishio, Physica C 279, 241 (1997).

57 K. Nakao, Yu. Eltsev, J. G. Wen, S. Shibata, and N. Koshizuka, Physika C, 322, 79 (1999).

58 T. Fujii, I. Terasaki, T. Watanabe, and A. Matsuda, condmat/0205121

H9 Claus, G. W. Crabtree, K. G. Vandervoort, J. Z. Liu, D. Lam, E. D. Bukowski, and D. M. Ginsberg, J. Appl. Phys. 87, 5049 (1990).

60 A. Bertinotti, V. Viallet, D. Colson, J.-F. Marucco, J. Hammann, G. Le Bras, A Forget, Physica C 268, 257 (1996).

61 Terukazu Nishizaki, Kenji Shibata. Takahiko Sasaki, and Norio Kobayashi, Physica C 341-348, 957 (2000)

62 N. Kobayashi, T. Nishizaki, K. Shibata, T. Sato, M. Maki, and T. Sasaki, Physica C 362, 121 (2001).

63 M. Reedyk, C. V. Stager, T. Timusk, J. S. Xue, and J. E. Greedan, Phys. Rev. B 44, 4539 (1991).

${ }^{64}$ Kayuo Kadowaki and Kazuhiro Kimura, Phys. Rev. B 57, 11674 (1998)

65 Ichiro Matsubara, Ryoji Funahashi, Kazuo Ueno, Hirishi Yamashita, and Tomoji Kawai, Physica C 256, 33 (1996).

66 A. Junod, K.-W, Wang, T. Tsukamoto, G. Triscone, B. Revaz E. Walker, and J. Muller, Physica C 229, 209 (1994).

67 M. Y. Cheon, G. C. Kim, B. J. Kim, and Y. C. Kim, Physica C 302, 215 (1998).

68 Mun-Seog Kim, Sung-Ik Lee, Seong-Cho Yu, Irina Kuzemskaya, Efim S. Itskevich, and K. A. Lokshin, Phys. Rev. B 57, 6121 (1998).

69 J. R. Thompson, D. K. Christen and J. G. Ossandon, Physica B 194-196, 1557 (1994).

70 A. Maignan, C. Martin, V. Hardy, and Ch. Simon, Physica C 228, 323 (1994).

71 G. Villarad, A. Daignere, D. Pelloquin, A. Maignan, Physica C 314, 196 (1999). 